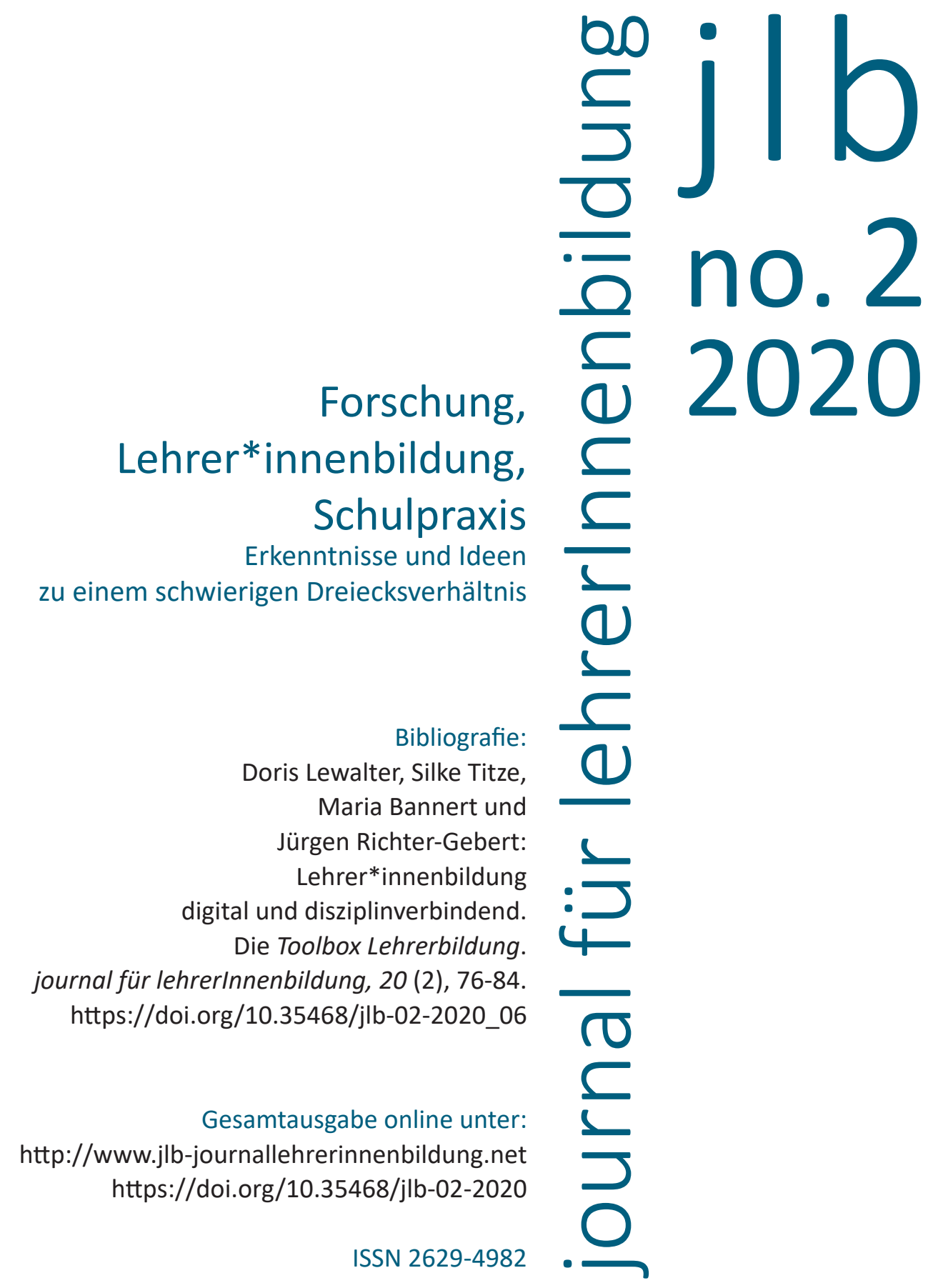


06

Doris Lewalter, Silke Titze, Maria Bannert und Jürgen Richter-Gebert

\section{Lehrer*innenbildung digital und disziplinverbindend. \\ Die Toolbox Lehrerbildung}




\section{Ausgangsüberlegungen}

Seit 2015 werden im Rahmen der Qualitätsoffensive Lehrerbildung im Projekt Teach@TUM, der TUM School of Education an zentralen Handlungsfeldern der Lehrer*innenbildung Verbesserungen vorgenommen. Hauptanliegen des Projekts ist die Qualitätsentwicklung der Lehrer*innenbildung durch eine Intensivierung der Abstimmung von Fach, Fachdidaktik, Erziehungswissenschaft und Schulpraxis (Seidel, Reiss, Bauer, Bannert, Blasini, Hubwieser, Jurik, Knogler, Lewalter, Nerdel, Riedl \& Schindler, 2016). Dazu werden u. a. Lehr-Lern-Materialien entwickelt, die auf eine praxisorientierte Kompetenz- und Evidenzbasierung der Ausbildung abzielen und an andere Standorte und Institutionen aller Phasen der Lehrer*innenbildung disseminiert werden können. Ein Produkt ist die Toolbox Lehrerbildung, die im Folgenden vorgestellt wird.

Den Ausgangspunkt für die Entwicklung der Toolbox Lehrerbildung bildet der häufig kritisierte Mangel an Vernetzung zwischen den an der Lehramtsausbildung beteiligten Disziplinen. So sieht Blömeke (2009) in der weitgehend unverbundenen Ausbildung in den drei zentralen Pfeilern der universitären Lehrer*innenbildung Erziehungswissenschaft/Psychologie, Fachdidaktik und Fachwissenschaft einen wesentlichen Grund für den defizitären Bezug der Lehramtsausbildung zur Schulpraxis. Von diesem Defizit ausgehend wird aufbauend auf dem Vernetzungsansatz der COACTIV-Studie (Baumert \& Kunter, 2006) in der Toolbox Lehrerbildung Wissen zu den drei Disziplinen anhand eines konkreten Unterrichtsbezugs vernetzt dargeboten (Lewalter, Schiffhauer, Richter-Gebert, Bannert, Engl, Maahs, Reißner, Ungar \& von Wachter, 2018). Dazu wird auf die vielfältigen Präsentations- und Gestaltungsmöglichkeiten digitaler Medien zurückgegriffen. Ihr Einsatz erlaubt es, eine instruktionale und informatorische Lernumgebung zu schaffen, mit deren Hilfe (angehende) Lehrpersonen in Studium und Referendariat flexibel praxisorientierte Kompetenzen erwerben können, indem Lehr-Lernprozesse berufsfeldbezogen gestaltet und ein authentisches, fallbezogenes sowie individualisiertes Lernen ermöglicht wird (Petko \& Honegger, 2001). 


\section{Aufbau und mediale Komponenten}

Die Toolbox Lehrerbildung ist eine online basierte, öffentlich zugängliche und kostenlose Lehr- und Lernplattform (www.toolbox.edu.tum.de). Der inhaltliche Aufbau orientiert sich an Lehr-Lern-Modulen. Innerhalb eines Moduls wird jeweils ein Thema aus jeder Disziplin aufgearbeitet, woraus sich für jedes Modul ein thematisches Dreigespann aus Fachwissenschaft - aktuell vor allem Mathematik -, Fachdidaktik und Erziehungswissenschaft/Psychologie ergibt (siehe Abb. 1). Dabei werden jeweils zentrale Themen und Fragestellungen aufbereitet, die am Bedarf der Lehrenden in der ersten Phase der Lehrer*innenausbildung ausgerichtet sind. Die Themenauswahl orientiert sich an Grundlagenthemen der jeweiligen Disziplin, die zum einen eine hohe und dauerhafte Relevanz und zum anderen eine evidenzbasierte Absicherung aufweisen.

\begin{tabular}{ccc}
$\begin{array}{c}\text { Erziehungswissenschaft/ } \\
\text { Psychologie }\end{array}$ & Fachdidaktik & Fachwissenschaft \\
\hline $\begin{array}{c}\text { Feedback } \\
\text { Aktivierung }\end{array}$ & $\begin{array}{c}\text { Beweisen und } \\
\text { Argumentieren }\end{array}$ & $\begin{array}{c}\text { Der Satz des } \\
\text { Pythagoras }\end{array}$ \\
\hline $\begin{array}{c}\text { Lehren und Lernen } \\
\text { mit digitalen Medien }\end{array}$ & Problemlösen & $\begin{array}{c}\text { Dreiecks- und } \\
\text { Quadratzahlen }\end{array}$ \\
\hline $\begin{array}{c}\text { Heterogenität und } \\
\text { adaptiver Unterricht }\end{array}$ & Didaktische Prinzipien & $\begin{array}{c}\text { Sinus, Cosinus \& } \\
\text { ihre Ableitungen }\end{array}$ \\
\hline $\begin{array}{c}\text { Kognitive Aktivierung } \\
\text { Lernbegleitung und } \\
\text { Lerncoaching }\end{array}$ & Rolle von Fehlern & Algorithmik \\
\hline Unterrichtsplanung & Ebenen der Repräsentation & Bruchrechnen \\
\hline
\end{tabular}

Abb. 1 Lehr-Lernmodule der Toolbox Lehrerbildung (Stand: Juni 2020)

Der Aufbau der Toolbox Lehrerbildung basiert auf der Idee eines Baukastens. Die Lehr-Lernmodule bestehen aus einer Zusammenstellung verschiedener medialer Komponenten, die einzeln oder in Kombination genutzt werden können und es erlauben, sich den Themen auf verschiedene Art und Weise zu nähern und sich mit ihnen auseinan- 
derzusetzen (siehe Abb. 2). Damit zielt die Gestaltung der Materialien der Toolbox Lehrerbildung auf eine größtmögliche Flexibilität in der Nutzung der einzelnen Inhalte ab.

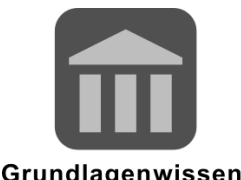

Grundlagenwissen

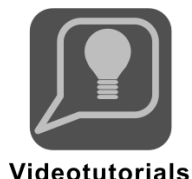

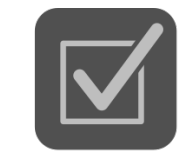

Lernaufgaben

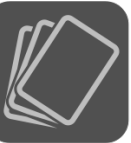

Begleitmaterialien \& Anwendungsszenarien

Abb. 2 Mediale Komponenten der Toolbox Lehrerbildung

In Grundlagentexten wird die aktuelle Fachliteratur sowohl zu theoretischen Konzeptionen als auch zum aktuellen Stand der Forschung aufbereitet. Dabei werden u. a. wissenschaftliche Evidenzen zu den jeweiligen theoretischen Annahmen vorgestellt sowie Forschungsbefunde zur unterrichtlichen Realität berichtet. Damit zielt die Toolbox Lehrerbildung auf eine evidenzbasierte Auseinandersetzung mit zentralen Themen des Lehrens und Lernens ab. Videotutorials dienen dazu, komplexe theoretische Konzeptionen Schritt für Schritt zu entwickeln und anschaulich vorzustellen. In Form von gescripteten Unterrichtsvideos wird die Schulwirklichkeit als praxisnaher disziplinverbindender Anker genutzt und explizit in den Lern- und Vermittlungsprozess eingebunden. Theoretische, wissenschaftliche und praxisorientierte Perspektiven und Evidenzen der drei beteiligten Disziplinen bilden die Basis für die Entwicklung der Drehbücher für die gescripteten Sequenzen der Unterrichtsvideos. Mithilfe der Unterrichtsvideos können Theorie und wissenschaftliche Evidenz realitätsnah auf professionsorientierte Situationen bezogen und eng miteinander verknüpft werden. Der Einsatz gescripteter Unterrichtsvideos ermöglicht es, in sehr konzentrierter Form vielfältige Formen der Umsetzung der Inhalte aller drei Disziplinen im Schulkontext darzustellen. Dynamische mathematische Visualisierungen dienen der Veranschaulichung und Vermittlung von 
Fachinhalten aus Mathematik und Informatik auf unterschiedlichen Anforderungsniveaus. Sie können, je nach Komplexität, sowohl für die Vermittlung im Lehramtsstudium als auch im Schulunterricht eingesetzt werden und werden für den Download zur Verfügung gestellt. Eine weitere Komponente stellen die sowohl disziplinspezifischen als auch disziplinverbindenden (Lern-)Aufgaben dar, die in Anlehnung an die Merkmale "Austausch", "Perspektivenwechsel", "Transferfragen" und „praktische Fragen“ konzipiert werden (Krammer \& Reusser, 2005). Hierbei werden bei manchen Aufgaben die Videosequenzen in die Aufgabenstellung einbezogen. Die Aufgaben sind nach Einsatzgebiet gegliedert. In jedem Modul finden sich Aufgaben für das Selbststudium, für den Einsatz im Unterricht und für den Einsatz in der Hochschule.

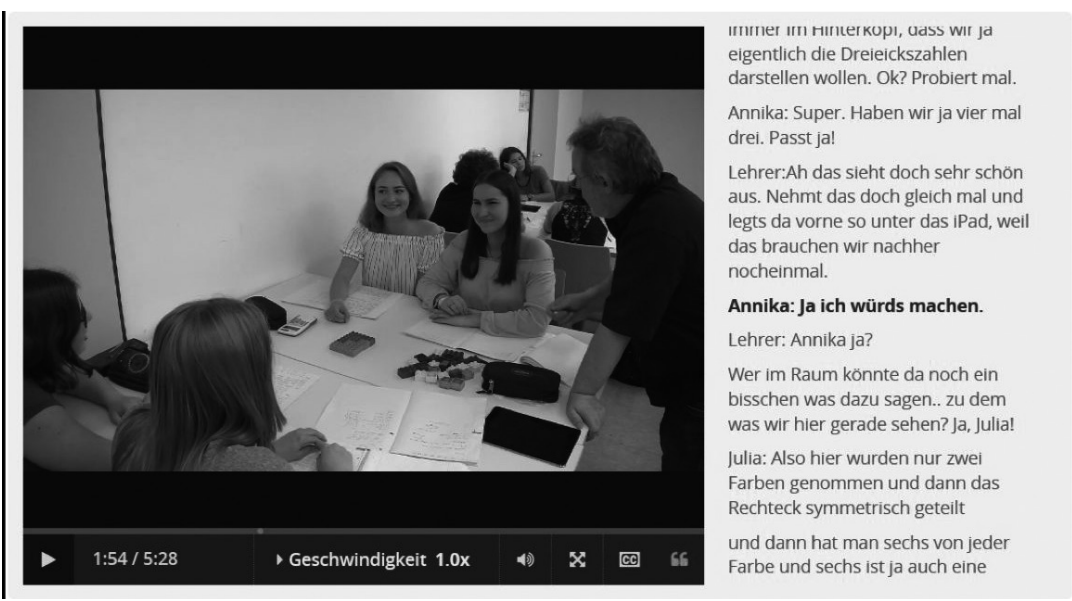

Abb. 3 Exemplarischer Screenshot eines Videos aus der Toolbox Lehrerbildung

Für Lehrende werden Begleitmaterialien zur Verfügung gestellt, die grundlegende Informationen, u. a. zum Einsatz von Videos in der Lehrer*innenbildung oder Visualisierungen im Lehr-Lern-Geschehen bereitstellen, aber auch konkrete Anregungen und Hilfestellungen für den Einsatz der medialen Komponenten der Plattform und ihre Kombination in unterschiedlichen instruktionalen Settings bieten. Zudem werden die Unterrichtsverlaufspläne zu den gescripteten Unterrichtsvideos zur Verfügung gestellt, die auch als Handlungsanleitung für Referendare und Lehrpersonen dienen. 


\section{Erprobte Einsatzgebiete}

Die Entwicklung der Toolbox Lehrerbildung ist eng mit einem elaborierten Evaluationskonzept verbunden. Dieses umfasst ein anfängliches Prototyping der Lehr-Lernmodule, bei dem verschiedene Nutzergruppen (Studierende und Dozierende) gebeten werden, sich mit der Plattform zu beschäftigen und anschließend an einem leitfadengestützten Einzelinterview teilzunehmen, in welchem die Lernplattform und ihre Komponenten, sowie deren Nutzungsmöglichkeiten und etwaige Kritikpunkte und Verbesserungsvorschläge besprochen werden. Die Befunde des Prototyping fließen in die Optimierung und (Weiter-)Entwicklung der Module ein. Dem Prototyping folgen formative und summative Evaluationen in Lehrveranstaltungen, sowie die implizite Erfassung von Nutzerdaten. Die Durchführung von bislang neun formativen Evaluationen in unterschiedlichen Lehrveranstaltungen an der TUM School of Education und sechs summativen Evaluationen an Hochschulen in Deutschland ergab sehr positive Einschätzungen hinsichtlich Usability und Akzeptanz auf Seiten der Lernenden. Ebenso zeigten Skalen zu Motivation und Interesse durchgehend gute Werte. Bei der oben bereits angesprochenen prototypischen Anwendung der Toolbox Lehrerbildung in zwei vergleichbaren Flipped Classroom Szenarien wurde, wie in allen weiteren Evaluationen, bei den Lehramtsstudierenden ein signifikanter Lerngewinn erzielt. Dieser bezieht sich insbesondere auf das Erkennen und Charakterisieren, aber auch auf das Beschreiben relevanter Unterrichtsmerkmale sowie die Vorhersage von deren Effekten. Bedeutsame Einflüsse der Arbeit mit der Toolbox Lehrerbildung auf die Entwicklung und Reflexion von Unterricht sind vor allem nach einer intensiveren Nutzung der Lernplattform erkennbar. Dieses iterative Vorgehen bei der wissenschaftlichen Begleitung trägt zu einem evidenzbasierten Entwicklungsprozess der Inhalte und Materialien bei.

\section{Nutzungsformen}

Lehrerbildende aller Phasen können die Inhalte und Materialien der Toolbox Lehrerbildung, entsprechend eines Baukastens in vielfältiger Weise, selektiv und flexibel nutzen, um sie in ihren Lehrveranstaltungen (Vorlesungen, Seminare, Übungen) einzusetzen oder ihre Stu- 
dierenden dazu anzuregen, sich Themen eigenständig anzueignen. Dies kann sowohl disziplinspezifisch, aber auch vernetzt über zwei oder drei Disziplinen hinweg erfolgen. Dabei können unterschiedliche instruktionale Ansätze zum Einsatz kommen, wie u. a. die direkte Instruktion in Form von Informationsinput anhand der Inhalte der Plattform oder Flipped Classroom Szenarien, bei denen Materialien der Plattform für die individuelle Vorbereitung der Plenumsveranstaltung verwendet werden. So wurden beispielsweise zur Förderung einer professionellen Unterrichtswahrnehmung (u. a. van Es \& Sherin, 2002) zur Vorbereitung einer Lehrveranstaltung Grundlagentexte zu verschiedenen Motivationstheorien von den Studierenden im Selbststudium bearbeitet. In der darauffolgenden Seminarsitzung wurden diese rekapituliert, bevor Videosequenzen zunächst in Zweiergruppen und dann im Plenum, bezogen auf das Erkennen (oder Feststellen) von motivational relevanten Unterrichtsmerkmalen, analysiert (noticing) wurden. Anschließend wurden die Videosequenzen hinsichtlich Handlungsalternativen auf der Basis des Unterrichtsgeschehens und der jeweils zugrundeliegenden theoretischen Konzepte reflektiert (reasoning). In anderen Veranstaltungen kamen die (Lern-)Aufgaben der Plattform zur gemeinsamem Bearbeitung in Kleingruppen zum Einsatz oder es wurden Videotutorials als Einführung in ein Thema genutzt.

Da die Toolbox Lehrerbildung zunehmend an verschiedenen Standorten der Lehrer*innenbildung im deutschsprachigen Raum eingesetzt wird, sind zum einen Angebote zur Einführung in die Nutzung der Plattform und zum anderen Angebote zum Erfahrungsaustausch bzw. zur Vernetzung von Dozierenden geplant. Entsprechende Informationen werden unter www.toolbox.edu.tum.de/aktuelles bekannt gegeben. 


\section{Literatur}

Baumert, J. \& Kunter, M. (2006). Stichwort: Professionelle Kompetenz von Lehrkräften. Zeitschrift für Erziehungswissenschaft, 9 (4), 469-520.

Blömeke, S. (2009). Lehrerausbildung. In S. Blömeke, T. Bohl, L. Haag, G. Lang-Wojtasik \& W. Sacher (Hrsg.), Handbuch Schule. Theorie - Organisation - Entwicklung (S. 483-490). Bad Heilbrunn/Stuttgart: Klinkhardt/UTB.

Krammer, K. \& Reusser, K. (2005). Unterrichtsvideos als Medium der Aus- und Weiterbildung von Lehrpersonen. Beiträge zur Lehrerbildung, 23 (1), 35-50.

Lewalter, D., Schiffhauer, S., Richter-Gebert, J., Bannert, M., Engl, A.-T., Maahs, M., Reißner, M., Ungar, P. \& von Wachter, J.-K. (2018). Toolbox Lehrerbildung - berufsfeldbezogene Vernetzung von Fach, Fachdidaktik und Bildungswissenschaft. In I. Glowinski, A. Borowski, J. Gillen, J. von Meien \& S. Schanze (Hrsg.), Kohärenz in der Universitären Lehrerbildung. Vernetzung von Fachwissenschaft, Fachdidaktik und Bildungswissenschaften (S. 331-353). Potsdam: Universitätsverlag Potsdam.

Petko, D. \& Honegger, B. (2011). Digitale Medien in der schweizerischen Lehrerinnenund Lehrerbildung: Hintergründe, Ansätze und Perspektiven. Beiträge zur Lehrerinnen- und Lehrerbildung, 29 (2), 155-171.

Seidel, T., Reiss, K., Bauer, J., Bannert, M., Blasini, B., Hubwieser, P., Jurik, V., Knogler, M., Lewalter, D., Nerdel, C., Riedl, A. \& Schindler, C. (2016). Kompetenzorientierte und evidenzbasierte Lehrerinnen- und Lehrerbildung: Didaktische Weiterentwicklungen im Projekt Teach@TUM. Beiträge zur Lehrerinnen- und Lehrerbildung, 34 (2), 230-242.

van Es, E. A. \& Sherin, M. G. (2002). Learning to Notice: Scaffolding New Teachers' Interpretations of Classroom Interactions. Society for Information Technology \& Teacher Education, 10 (4), 571-596. 


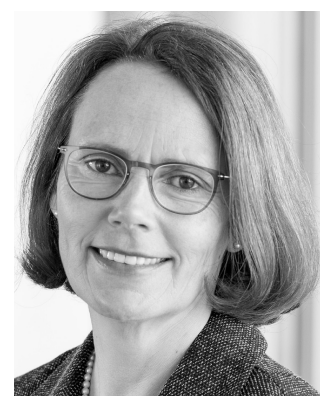

Doris Lewalter, Dr. Prof.,

Professur für Formelles und Informelles Lernen,

TUM School of Education.

Arbeitsschwerpunkte:

Lehren und Lernen in formellen und

informellen Kontexten,

Motivationsforschung, Evaluation

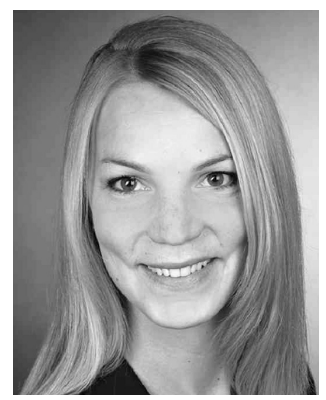

doris.lewalter@tum.de

Silke Titze, Dr., Projektkoordinatorin im Teach@TUM, TUM School of Education.

Arbeitsschwerpunkt:

Toolbox Lehrerbildung

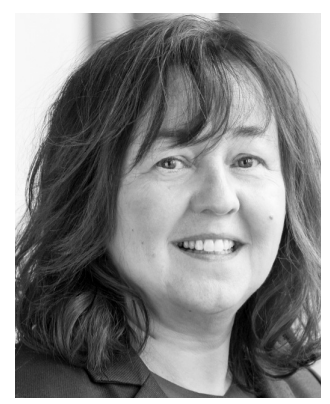

silke.titze@tum.de

Maria Bannert, Dr. Prof.,

Lehrstuhl Lehren und Lernen mit Digitalen Medien, TUM School of Education.

Arbeitsschwerpunkte:

Selbstreguliertes Lernen, Digitale Lehr-Lern-Medien,

Lernprozessanalysen

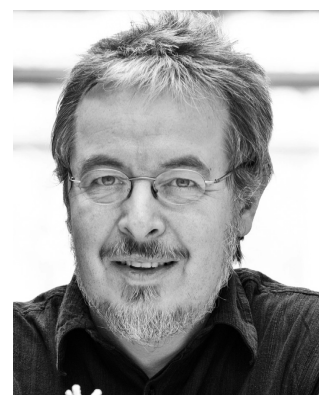

maria.bannert@tum.de

Jürgen Richter-Gebert, Dr. Prof., Zentrum Mathematik TU München.

Arbeitsschwerpunkte:

Geometrie und Visualisierung, computergestützte Mathematik, Mathematikvermittlung in der Öffentlichkeit

richter@ma.tum.de 